\title{
The Clinical Utility of Kinetic Glomerular Filtration Rate in the Assessment of Renal Function and Prediction of Outcomes Among Critically III Patients With \\ Acute Kidney Injury:
A Single-Center Retrospective \\ Acute Kidney Injury:
A Single-Center Retrospective Cohort Study
} $\$$

Shari Ann Atanacio, MD, Maria Rachel Uy, MD

\section{ABSTRACT}

Objective: To determine the discriminatory ability of kinetic glomerular filtration rate (kGFR) to detect acute kidney injury (AKI) when compared with established GFR equations and criteria and relating it to mortality, renal replacement therapy initiation and renal recovery.

Methods: This was a retrospective analysis using data from chart review of 109 intensive care unit (ICU) patients at the University of Santo Tomas Hospital (USTH). The renal function estimates using Chronic Kidney Disease Epidemiology Collaboration (CKDEpi), modification of diet in renal disease (MDRD), Kidney Disease Improving Global Outcomes Acute Kidney Injury (KDIGO AKI), as well as kinetic GFR equations were compared and correlated with renal and cardiovascular outcomes.

Results: The renal function assessed by kGFR, CKD-Epi, MDRD and KDIGO staging based on serum

Shari Ann Atanacio

shari.atanacio@gmail.com

Section of Adult Nephrology, University of Santo Tomas Manila, Philippines creatinine $(\mathrm{SCr})$ showed no significant association with mortality outcomes. However, AKI diagnosed based on urine output (UO), and combined SCr and urine output (KDIGO) showed association with allcause mortality. The UO detected severe stages of AKI while $\mathrm{SCr}$ (based on KDIGO) better identified the earlier stages of AKI. The criteria for KDIGO AKI when combined also shows mortality prediction since it joins together the effects of $\mathrm{SCr}$ and $\mathrm{UO}$. There was a remarkable 3.5 times increase in hemodialysis initiation $(p=0.0001)$ and 12.89 times increase in peritoneal dialysis initiation $(p=0.01)$ for every stage increase in the KDIGO classification. kGFR, CKD-Epi and MDRD have 5\%, 6\%, and $6 \%$ decrease, respectively in the odds of initiating hemodialysis. There was however, no association for peritoneal dialysis.

Conclusion: kGFR was the least able in detecting $A K I$ and KDIGO AKI criteria remains to be the standard in identifying $A K I$ in the critical care setting. Increase in $\mathrm{SCr}$ was a sensitive tool in diagnosing $\mathrm{AKI}$ due to its ability to detect AKI based on a small increase in $\mathrm{SCr}$ regardless of the baseline renal function. Decreasing UO, however, is the prognosticating variable in KDIGO AKI criteria, 
in that it portends higher probability of initiation of renal replacement therapy (RRT) and ultimately higher mortality when present.

\section{INTRODUCTION}

Acute kidney injury (AKI) is a common cause of morbidity and mortality among critically ill patients with incidence varying from $36 \%$ to $67 \%$. [1] It is shown that variation in serum creatinine ( $\mathrm{SCr}$ ) is associated with poor outcomes in hospitalized patients.[2] It is however challenging to assess an acutely unwell patient with rapidly changing kidney function.

The commonly used formulae such as the Modification of Diet in Renal Disease (MDRD) or chronic kidney disease epidemiology collaboration (CKD-Epi) are used in patients with stable renal function to compute the estimated glomerular filtration rate (eGFR). Unfortunately, these current formulae fall short in an acutely unwell patient with rapidly changing renal function.

The current approaches to classify AKI severity and identify patients at the highest risk for poor outcomes focus on the maximum change in $\mathrm{SCr}$ values. The non-steady-state kinetic eGFR could add clinical and prognostic information in critically ill patients beyond the current AKI classification system.

In critically ill patients with AKI, it is imperative that we have an estimate of their renal function for the proper adjustment of medication dosages, nutrition and need for renal replacement therapy (RRT). These measures must be instituted at the earliest possible time to minimize unwanted outcomes. kGFR takes into account these shortcomings and reinforces the relevance of timely detection of impending AKI. If proven to be accurate in unstable renal function estimation among critically ill patients, its clinical utility lies in secondary preventive measures that will subsequently limit AKI progression, thereby increasing the recovery chances of our patients.

There is no universally accepted and validated measure of renal function among patients with AKI, more so among critically ill patients. In this subset of patients, the estimates of kidney function are vital to adjustments in drug dosing, revision of nutritional therapy and decisions to provide dialytic support. However, estimating GFR is challenging due to fluctuations and instabilities in kidney function, creatinine production and fluid balance. The common tools in estimating GFR (ie, CKD-Epi, MDRD) are only used when kidney function and $\mathrm{SCr}$ are stable. This led to recent efforts to quantify and classify AKI based on changes in $\mathrm{SCr}$ and diuresis. Kidney Disease Improving Global Outcomes (KDIGO) developed the $\mathrm{AKI}$ criteria in an attempt to standardize the definition and risk stratification of susceptible patients. [3] This widely used criteria categorized AKI in stages but failed to provide a precise measurement of the kidney function. Potential piffalls of the criteria include the need for a minimum timeframe ( $48 \mathrm{~h}$ ) to proceed with the classification and its inconsideration of baseline renal function level (history of chronic kidney disease) and rapidity of renal function deterioration. This inevitable time window can be a potential cause of delay in the detection and management of AKI.

Kinetic glomerular filtration rate (kGFR) was developed by Chen to analyze the renal function during AKI.[4] It is derived from the initial creatinine content, volume of distribution, creatinine production rate and the quantitative difference between consecutive SCrs over a given time. For that period, the computed creatinine excretion then yields the creatinine clearance rate. Additional formula variables needed in the computation are any steadystate plasma creatinine, the corresponding eGFR by an empirical formula, and the maximum increase in creatinine per day if the patient is anuric. The kinetic formula guides the nephrologists in estimating renal function and complements their clinical intuition by adding a quantitative value to rapidly changing $\mathrm{SCr}$ during AKI and in the recovery phase.

The calculation of kGFR also facilitates early prediction of delayed graft function among renal transplant patients within 4 hours of renal transplantation.[5] In an acute critical setting, kGFR was noted to be significantly associated with AKI and initiation of renal replacement therapy (RRT) compared to MDRD but failed to correlate with 30day mortality and cardiovascular outcomes. [6] There have been no studies addressing the relationship of kGFR and other parameters to renal outcomes in terms of recovery and maintenance of RRT.

The general objective of the study was to determine the clinical utility of kGFR in assessing renal function and correlating outcomes of critically ill patients with AKI. Specifically, it compared the estimates of renal function during $A K I$ using the different parameters of KDIGO-AKI criteria, CKD-Epi, MDRD formula 
and KGFR and their correlation to the following endpoints: (a) all cause mortality, (b) initiation of RRT and (c) resolution of AKI. This study also determined the sensitivity of kGFR in assessing AKI among critically ill patients.

\section{METHODOLOGY}

Patients admitted to the UST Hospital Cardiovascular Unit (CVU) and Critical Care Unit (CCU) from January 2017 to December 2017 were included in the study. A chart review of the admissions was done and appropriate data collected using an abstraction form (see appendix) and a retrospective cohort analysis design employed. For patients with multiple CCU/ CVU admissions for the same condition, we only included the initial critical admission and treated the subsequent admissions as outcomes.

Patient profiles included patients greater than 18 years of age, ICU stay of more than $48 \mathrm{~h}$ with at least two SCr measurements taken (prior to RRT for those who underwent dialysis) during admission. Patients excluded were known cases of end-stage renal disease, renal transplant patients, transferred patients from other institutions and those who underwent RRT before onset of critical condition.

A sample size of 109 patients yielded a power of $80.00 \%$ at a significance level of $5.00 \%$ (two-tailed). $[9,10]$ Data extracted from chart review included demographic profile (age, gender), comorbidities, admission data and mortality outcomes. In addition, clinical data collected included but not limited to clinical diagnosis, clinical status (with requirements for vasoactive drugs or mechanical intubation, development of oliguria or anuria, decline in sensorium, etc.), surgical interventions, laboratory and ancillary results.

Patients' admission $\mathrm{SCr}$ was treated as the baseline, unless an accessible $\mathrm{SCr}$ within three months prior to admission was available. If during the course of admission $\mathrm{SCr}$ eventually went down, the nadir $\mathrm{SCr}$ was reconsidered as the baseline. Disease severity based on APACHE II score was also computed if available data permitted. Serial $\mathrm{SCr}$ measurements were used as a gauge for renal function when using kinetic eGFR formula. This was likewise the case for estimation of kidney function when using the KDIGO-AKI criteria together with the recorded urine output. The highest recorded $\mathrm{SCr}$ was used to estimate renal function when using CKDEpi and MDRD formula. The worst recorded KDIGOAKI, CKD Epi, MDRD and kGFR levels during the course of admission or prior to initiation of RRT (for those who underwent dialysis) was used.

All-cause mortality was set as the primary endpoint of the study. Secondary endpoints included initiation of RRT (hemodialysis, peritoneal dialysis, continuous RRT). Those who underwent RRT were further evaluated in terms of AKI resolution. The following operational definitions of AKI resolution were used: (a) no resolution - if a patient was dialysis dependent upon discharge or mortality; (b) partial resolution - weaned off from RRT but $\mathrm{SCr}$ failed to go back to normal, nadir or baseline; (c) complete resolution - weaned off from RRT with $\mathrm{SCr}$ going back to normal, or $\mathrm{SCr}$ decreased within $25 \%$ of nadir or baseline.

Patients who developed AKI but were not initiated on RRT were also assessed on the same outcomes as those in the RRT group: (a) no resolution - discharge $\mathrm{SCr}$ is the same, higher or fails to decrease by $>50 \%$ from zenith $\mathrm{SCr}$ (highest recorded $\mathrm{SCr}$ during admission); (b) partial resolution - discharge $\mathrm{SCr}$ decreased by $>50 \%$ from zenith $\mathrm{SCr}$ but failed to go back to normal, nadir or baseline $\mathrm{SCr}$; (c) complete resolution - discharge $\mathrm{SCr}$ decreased going back to normal, or $\mathrm{SCr}$ decreased within $25 \%$ of nadir or baseline SCr.

A Cox regression for survival analysis was performed to assess survival by AKI severity. Logistic regression analysis was used to assess the association and effect of renal parameters (kGFR, KDIGO, eGFR) in predicting prespecified outcomes. Receiver operating characteristic ( $R O C$ ) analysis was used to find the optimal cut-point value for each assessment that best discriminates between selected prespecified outcomes. Each ROC analysis included an ROC curve, a table with the sensitivities and specificities at certain assessment cut-points, the area under the ROC curve (AUC) and 95\% confidence intervals (Cls). AUC curves were compared using the Delong method. The significance threshold was set at 0.05 for all calculations.

\section{RESULTS}

The study population included 109 admitted critical care patients. Table 1 shows the demographic profile 
Table 1. Demographic Profile of Patients $(N=109)$

\begin{tabular}{|c|c|c|c|}
\hline Characteristic & Frequency (f) & $\%$ & Mean (SD) or Median (IQR) \\
\hline Age & & & $68.62(14.04)$ \\
\hline \multicolumn{4}{|l|}{ Sex } \\
\hline Male & 63 & $57.80 \%$ & \\
\hline Female & 46 & $42.20 \%$ & \\
\hline \multicolumn{4}{|l|}{ Renal Diagnosis } \\
\hline AKI due to Sepsis & 50 & $45.87 \%$ & \\
\hline AKI due to ATN & 1 & $0.92 \%$ & \\
\hline AKI due to CRS & 34 & $31.19 \%$ & \\
\hline AKI due to Obstructive Uropathy & 2 & $1.83 \%$ & \\
\hline AKI due to Decreased ECV & 15 & $13.76 \%$ & \\
\hline AKI due to AIN & 2 & $1.83 \%$ & \\
\hline AKI due to Hepatorenal Syndrome & 1 & $0.92 \%$ & \\
\hline AKI due to Ischemic Nephropathy & 1 & $0.92 \%$ & \\
\hline AKI due to TTP & 1 & $0.92 \%$ & \\
\hline$A K I$ due to $D H N$ & 2 & $1.83 \%$ & \\
\hline \multicolumn{4}{|l|}{ CV Outcome } \\
\hline Discharged & 61 & $55.96 \%$ & \\
\hline Expired & 48 & $44.04 \%$ & \\
\hline \multicolumn{4}{|l|}{ Renal Outcome } \\
\hline No Resolution & 57 & $52.29 \%$ & \\
\hline Partial Resolution & 3 & $2.75 \%$ & \\
\hline Complete Resolution & 49 & $44.95 \%$ & \\
\hline \multicolumn{4}{|c|}{ Initiation of Renal Replacement Therapy (RRT) } \\
\hline No RRT & 60 & $55.05 \%$ & \\
\hline Hemodialysis & 41 & $37.61 \%$ & \\
\hline Peritoneal Dialysis & 8 & $7.34 \%$ & \\
\hline \multicolumn{4}{|l|}{ Underlying CKD } \\
\hline Yes & 66 & $60.55 \%$ & \\
\hline No & 43 & $39.45 \%$ & \\
\hline Number of Inotropes & & & $1(0-3)$ \\
\hline \multicolumn{4}{|l|}{ Invasive Ventilation } \\
\hline Yes & 48 & $44.04 \%$ & \\
\hline No & 61 & $55.96 \%$ & \\
\hline
\end{tabular}

AKI - acute kidney injury

ATN - acute tubular necrosis

CRS - cardiorenal syndrome

$E C V$ - effective circulating volume

AIN - acute interstitial nephritis

TTP - thrombotic thrombocytopenic purpura

$D H N$ - dehydration

of patients with the mean age of 68.62 (14.04). Most of them were male $(57.80 \%)$, had AKI due to sepsis $(45.87 \%)$, were discharged $(55.96 \%)$, had no renal resolution $(52.29 \%)$, had no RRT $(55.05 \%)$, and had underlying CKD (60.55\%). The median number of inotropes was 1 with an IQR of $0-3$. Most respondents did not have invasive ventilation (59.96\%). 
Table 2. Clinical and Renal Profiles of the Patients

Descriptive Statistics $(N=109)$

\begin{tabular}{lccc}
\hline Characteristic & Frequency (f) & $\%$ & Mean (SD) or Median (IQR) \\
\hline APACHE Score & & & $22.92(18.20)$ \\
Kinetic GFR & & & $24.51(14.29)$ \\
CKD-Epi & & & $24.69(13.87)$ \\
MDRD Score & & & \\
KDIGO Creatinine & 12 & $11.01 \%$ & \\
$\quad$ No AKI & 57 & $52.29 \%$ & \\
Stage 1 AKI & 23 & $21.10 \%$ & \\
Stage 2 AKI & 17 & $15.60 \%$ & \\
Stage 3 AKI & & & \\
KDIGO Urine Output & 49 & $44.95 \%$ & \\
No AKI & 8 & $7.34 \%$ & \\
Stage 1 AKI & 18 & $16.51 \%$ & \\
Stage 2 AKI & 34 & $31.19 \%$ & \\
Stage 3 AKI & & & \\
KDIGO & 3 & $2.75 \%$ & \\
No AKI & 32 & $29.36 \%$ & \\
Stage 1 AKI & 29 & $41.28 \%$ & \\
Stage 2 AKI & 45 & & \\
Stage 3 AKI & & & \\
\hline
\end{tabular}

The renal profile of these patients is presented in Table 2 showing the descriptive analysis for renal function tests. The mean APACHE score was 19.16 with approximate mortality of $24 \%$ for nonpostoperative and $12 \%$ for postoperative patients, respectively. [7] The study population mortality was $44 \%$, way above the expected mortality rate. The mean kGFR, CKD-Epi and MDRD scores were $22.92,24.51$ and 24.69, respectively. For the KDIGO creatinine, most had Stage 1 AKI (52.29\%) while the KDIGO urine output mostly had no AKI (44.95\%). The KDIGO AKI, with the combination of urine output and $\mathrm{SCr}$ as measures, mostly had Stage 3 AKI (41.28\%). KDIGO criteria of AKI had been considered the standard definition and classification of AKI. The criteria using solely the changes in $\mathrm{SCr}$ seemed to catch the earlier stages of AKI while the urine output detected more severe stages of AKI but when combined together increased the detection of all stages of AKI.

The mortality outcomes had no significant association with renal function assessed by kGFR, CKD-Epi, MDRD, KDIGO staging based on SCr. However, AKI diagnosed based on urine output, and combined $\mathrm{SCr}$ and urine output (KDIGO) showed association with all-cause mortality (see Table 3). The urine output detected severe stages of AKI while $\mathrm{SCr}$ (KDIGO) identified the early stage of AKI (see Table 2). Combining the criteria for KDIGO-AKI was also shown to be predictive of mortality since it combined together the effects of $\mathrm{SCr}$ and $\mathrm{UO}$, increasing the recognition of AKI.

There was a remarkable 3.5 times increase of initiating hemodialysis $(p=0.0001)$, and 12.89 times increase of initiating peritoneal dialysis $(p=0.01)$ for every stage increase in the KDIGO classification as shown in Table 4. Using renal estimates by KDIGO, the UO yielded 4.18 times increase for peritoneal dialysis initiation for every stage increase in the UO criteria. Similarly, individual indices for KDIGO have $71 \%$ and $215 \%$ increases in hemodialysis initiation for $\mathrm{SCr}$ and $\mathrm{UO}$ criteria, respectively. For kGFR, CKD-Epi, MDRD have 5\%,6\%, 6\% decrease, respectively in the odds of initiating hemodialysis. There was, however, no association for peritoneal dialysis.

Renal recovery was not associated with renal function estimated by KGFR, CKD-Epi and MDRD formula. In contrary, there was a significant decrease in the odds of complete recovery for every 
Table 3. Univariate Binary Logistic Regression of the Predictors of All-Cause Mortality $(N=109)$

Predictors

All-Cause Mortality

Odds Ratio Standard Error $\quad \begin{gathered}\text { p-values } \\ \text { (Two-tailed) }\end{gathered} \quad 95 \% \mathrm{Cl}$, Odds Ratio

\begin{tabular}{lllll}
\hline Kinetic GFR & 1.00 & 0.01 & 0.655 & $0.97,1.02$ \\
CKD-Epi & 1.00 & 0.01 & 0.982 & $0.97,1.03$ \\
MDRD Score & 1.00 & 0.01 & 0.780 & $0.97,1.03$ \\
KDIGO Creatinine & 1.49 & 0.34 & 0.076 & $0.96,2.32$ \\
KDIGO UO & 2.21 & 0.38 & $0.0001^{\dagger}$ & $1.58,3.09$ \\
KDIGO & 2.83 & 0.73 & $0.0001^{\dagger}$ & $1.71,4.70$ \\
\hline
\end{tabular}

†Significant at $\leq 0.01$ level

Table 4. Polynomial Logistic Regression of the Predictors of Renal Replacement Therapy ( $N=109$ )

Predictors Renal Replacement Therapy

Odds Ratio Standard Error $p$-values (Two-tailed) $95 \% \mathrm{Cl}$, Odds Ratio

Kinetic GFR

No RRT

Hemodialysis

1.00 (Referent)

Peritoneal Dialysis

$0.95^{\dagger}$

0.01

0.0001

$0.92,0.97$

0.96

0.02

0.101

$0.91,1.01$

CKD-Epi

No RRT

Hemodialysis

1.00 (Referent)

Peritoneal Dialysis

$0.94^{\dagger}$

0.02

0.001

$0.91,0.97$

0.99

0.03

0.695

$0.94,1.04$

MDRD Score

No RRT

Hemodialysis

Peritoneal Dialysis

KDIGO Creatinine

No RRT

Hemodialysis

Peritoneal Dialysis

KDIGO UO

No RRT

Hemodialysis

Peritoneal Dialysis

\section{KDIGO}

\section{No RRT}

Hemodialysis

Peritoneal Dialysis

* Significant at $\leq 0.05$ leve

${ }^{\dagger}$ Significant at $\leq 0.01$ level

GFR - glomerular filtration rate

$R R T$ - renal replacement therapy
1.00 (Referent)

$\begin{array}{llll}0.94^{\dagger} & 0.02 & 0.001 & 0.90,0.97 \\ 0.99 & 0.03 & 0.702 & 0.94,0.97\end{array}$

1.00 (Referent)

$1.71^{*}$

0.41

0.027

$1.06,2.74$

1.69

0.72

0.219

$0.73,3.88$

1.00 (Referent)

$\begin{array}{cccc}2.15^{\dagger} & 0.38 & 0.0001 & 1.52,3.05 \\ 4.18 & 2.03 & 0.003^{\dagger} & 1.61,10.82\end{array}$

1.00 (Referent)

\begin{tabular}{cccc}
$3.59^{\dagger}$ & 1.05 & 0.0001 & $2.03,6.35$ \\
$12.89^{\dagger}$ & 12.85 & 0.010 & $1.83,90.98$ \\
\hline
\end{tabular}

stage increase in KDIGO (OR 0.26, $p=0.0001$ ). Similarly, there was a significant reduction of complete recovery for every stage increase in $\mathrm{SCr}$ and $U O$ in the KDIGO criteria (OR 0.5, $p=0.006$; OR 0.42, $p=0.0001$, respectively) as shown in Table 5. 
Table 5. Polynomial Logistic Regression of the Predictors of Renal Outcome $(N=109)$

\begin{tabular}{|c|c|c|c|c|}
\hline \multirow[t]{2}{*}{ Predictors } & \multicolumn{4}{|c|}{ Renal Replacement Therapy } \\
\hline & Odds Ratio & Standard Error & p-values (Two-tailed) & $95 \% \mathrm{Cl}$, Odds Ratio \\
\hline \multicolumn{5}{|l|}{ Kinetic GFR } \\
\hline No Resolution & 1.00 (Referent) & - & - & - \\
\hline Partial Resolution & 1.04 & 0.03 & 0.177 & $0.98,1.10$ \\
\hline Complete Resolution & 1.02 & 0.01 & 0.115 & $1.00,1.04$ \\
\hline \multicolumn{5}{|l|}{ CKD-Epi } \\
\hline No Resolution & 1.00 (Referent) & - & - & - \\
\hline Partial Resolution & 1.07 & 0.04 & 0.089 & $0.99,1.15$ \\
\hline Complete Resolution & 1.01 & 0.01 & 0.545 & $0.98,1.04$ \\
\hline \multicolumn{5}{|l|}{ MDRD Score } \\
\hline No Resolution & 1.00 (Referent) & - & - & - \\
\hline Partial Resolution & 1.07 & 0.043 & 0.107 & $0.99,1.15$ \\
\hline Complete Resolution & 1.01 & 0.01 & 0.442 & $0.98,1.04$ \\
\hline \multicolumn{5}{|l|}{ KDIGO Creatinine } \\
\hline No Resolution & 1.00 (Referent) & - & - & - \\
\hline Partial Resolution & 1.04 & 0.69 & 0.947 & $0.29,3.78$ \\
\hline Complete Resolution & 0.50 & 0.13 & $0.006^{\dagger}$ & $0.31,0.82$ \\
\hline \multicolumn{5}{|l|}{ KDIGO UO } \\
\hline No Resolution & 1.00 (Referent) & - & - & - \\
\hline Partial Resolution & 0.55 & 0.27 & 0.220 & $0.21,1.42$ \\
\hline Complete Resolution & 0.42 & 0.08 & $0.0001^{\dagger}$ & $0.29,0.60$ \\
\hline \multicolumn{5}{|l|}{ KDIGO } \\
\hline No Resolution & 1.00 (Referent) & - & - & - \\
\hline Partial Resolution & 0.47 & 0.33 & 0.277 & $0.12,1.83$ \\
\hline Complete Resolution & 0.26 & 0.07 & $0.0001^{\dagger}$ & $0.15,0.45$ \\
\hline
\end{tabular}

*Significant at $\leq 0.05$ level

${ }^{\dagger}$ Significant at $\leq 0.01$ level

Comparison of different renal function tests in diagnosing $\mathrm{AKI}$ compared to KDIGO criteria, the current standard for definition and classification of AKI showed kGFR to be the least able to diagnose AKI (See Figure 1 and Table 6). Increase in $\mathrm{SCr}$ based on KDIGO criteria appeared to be the best diagnostic test due to its ability to detect early stages of $\mathrm{AKI}$ with small changes in $\mathrm{SCr}$, making it a sensitive measure of AKI. 


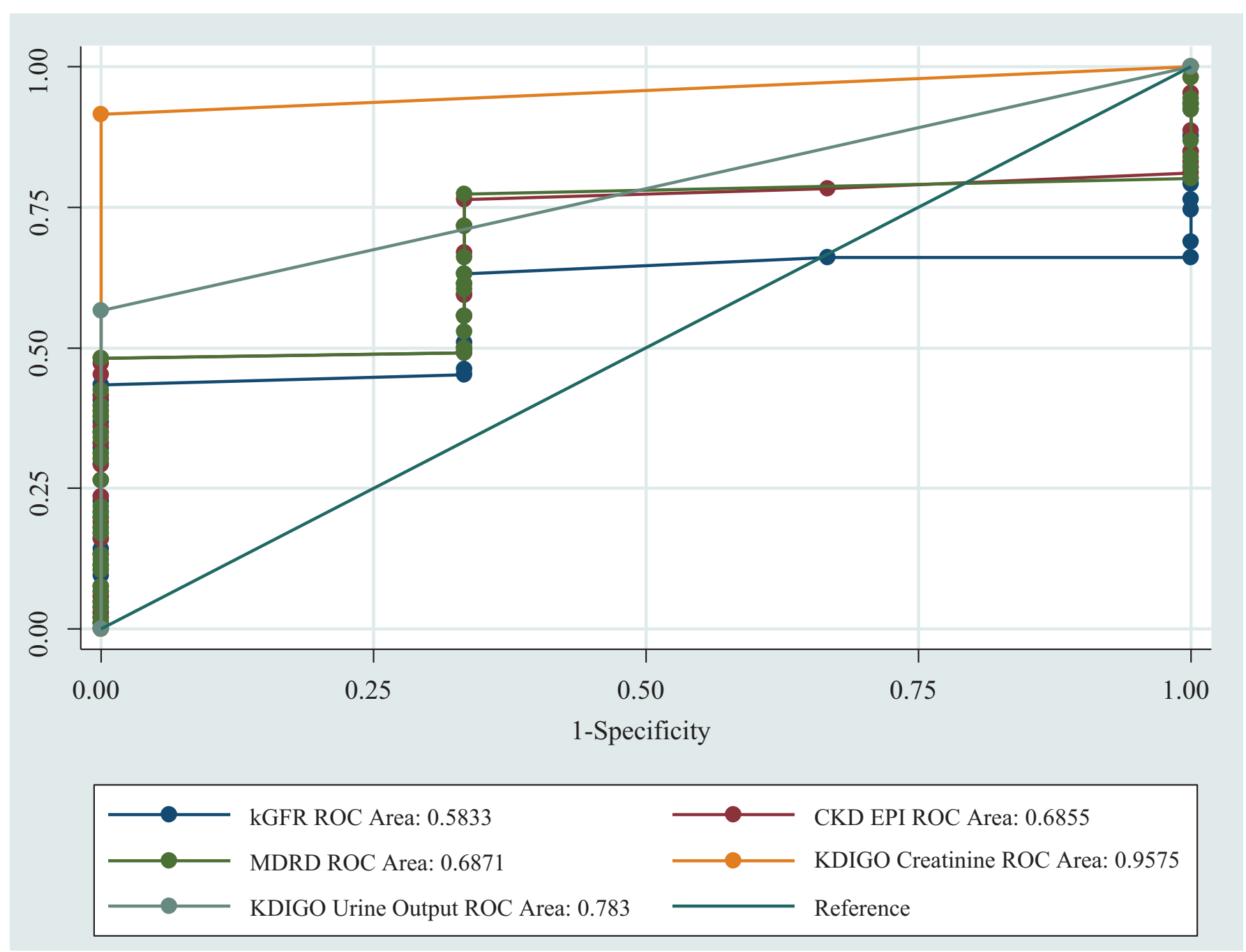

Figure 1. Receiver Operating Characteristics (ROC) of Renal Function against the Gold Standard (KDIGO

Table 6. Receiver Operating Characteristics (ROC) of Renal Function Against the Gold Standard (KDIGO) (N=109)

\begin{tabular}{lcccc}
\hline Renal Function & \multicolumn{5}{c}{} \\
\cline { 2 - 5 } & AUC & Standard Error & $\mathbf{9 5 \%} \mathbf{~ I ~}$ & p-value \\
\hline Kinetic GFR & 0.5833 & 0.08 & $0.42,0.74$ & 0.00001 \\
CKD-Epi & 0.6855 & 0.11 & $0.42,0.89$ & \\
MDRD Score & 0.6871 & 0.11 & $0.48,0.90$ & \\
KDIGO Creatinine & 0.9575 & 0.01 & $0.93,0.98$ & \\
KDIGO UO & 0.7830 & 0.02 & $0.74,0.83$ & \\
\hline
\end{tabular}

\section{DISCUSSION}

Equations used to estimate GFR like CKD-Epi and MDRD are often used in acute critical settings to estimate renal function despite the lack of data from studies supporting its validity in the said setting. These formulae provide physicians with renal function estimate for use of initial drug dosing, prescribed nutrition and even decisions whether to initiate dialysis. The problem with these equations is the tendency to overestimate GFR when the baseline $\mathrm{SCr}$ is low. [8] Small absolute changes in $\mathrm{SCr}$ will not be reflected as a significant relative change in GFR, particularly with a lower baseline SCr.

The above problem is taken into account by kGFR where it measures the change in $\mathrm{SCr}$ within a given time interval. Intuitively, the larger the excursion in the $\mathrm{SCr}$, the greater the effect will be on the kidney 
function. It is proportional to how much the GFR has improved or worsened in comparison with the steadystate GFR. The finding of kGFR inability to diagnose $A K I$ is secondary to the fact that some patients in the study were caught in the resolution phase of the AKI rather than during the initial kidney insult, hence the computed KGFR is higher and ultimately, it decreased its ability to detect AKI during the time of computation. The kGFR formula also incorporates the steady-state $\mathrm{SCr}$, the corresponding eGFR by an empirical formula and the maximum increase in creatinine per day if patient is anuric. If no steady-state $\mathrm{SCr}$ is available, the baseline $\mathrm{SCr}$ can be substituted as such. In this study, the admission $\mathrm{SCr}$ was taken as the steady-state $\mathrm{SCr}$ unless a preadmission $\mathrm{SCr}$ done within the last three months was available. The computed change in $\mathrm{SCr}$ was then compared to the baseline $\mathrm{SCr}$. If the admission $\mathrm{SCr}$ was high, then the change in $\mathrm{SCr}$ relative to the baseline was lower.

The KDIGO criteria combines both the increase in $\mathrm{SCr}$ and $\mathrm{UO}$ as parameters for AKI detection and classification. Increase in $\mathrm{SCr}$ based on KDIGO definition was a sensitive gauge in diagnosing AKI compared to the UO criteria due to minimal incremental increase in $\mathrm{SCr}(0.3 \mathrm{mg} / \mathrm{dl}$ within $48 \mathrm{~h})$ required. It was able to detect $\mathrm{AKI}$ the most among the renal function tests mostly on the earlier stage of AKI. UO was able to identify more severe stages of AKI, hence when UO became inadequate the tendency for initiation of RRT was higher with OR of 2.15 and 4.18 for HD and PD, respectively. Mortality also became significantly higher as UO became inadequate, whereas KGFR, CKD-Epi and MDRD did not reflect the same mortality and cardiovascular outcomes similar to the findings of O'Sullivan et al (2016). There was decreasing odds of complete renal recovery as the KDIGO stage became worse.

\section{CONCLUSION}

The KDIGO AKI criterion remains the standard in identifying AKI in the critical care setting. Increase in $\mathrm{SCr}$ was a sensitive tool in diagnosing AKI due to its ability to detect AKI based on a small increase in $\mathrm{SCr}$ regardless of baseline renal function. Decreasing urine output, however, is the prognosticating variable in KDIGO AKI criteria, in that it portends higher probability of initiation of RRT and ultimately higher mortality when present.

The researcher recommends utilizing prospective studies in AKI among subjects exclusively presenting with increasing azotemia. This research design will better gauge the clinical utility of kGFR diagnosing and prognosticating AKI. It is also preferable that long-term outcomes be measured. 


\section{REFERENCES}

1. de Oliveira Marques F, Oliveira SA, de Lima e Souza PF, Nojoza WG, da Silva Sena M, Ferreira TM, et al. Kinetic estimated glomerular filtration rate in critically ill patients: beyond the acute kidney injury severity classification system. Crit Care [Internet]. 2017 Nov 18;21(1). Available from: http://dx.doi.org/10.1186/s13054-017-1873-0

2. Chertow GM, Burdick E, Honour M, Bonventre JV, Bates DW. Acute Kidney Injury, Mortality, Length of Stay, and Costs in Hospitalized Patients. JASN [Internet]. 2005 Sep $21 ; 16(11): 3365-70$. Available from: http://dx.doi. org/10.1681/ASN.2004090740

3. Kellum JA, Lameire N, Aspelin P, Barsoum RS, Burdmann EA, Goldstein SL, et al. Kidney disease: Improving global outcomes (KDIGO) acute kidney injury work group. KDIGO clinical practice guideline for acute kidney injury. Kidney International Supplements. 2012 Mar;2(1):1-138. Available from: https://doi.org/10.1038/kisup.2012.1

4. Chen S. Retooling the Creatinine Clearance Equation to Estimate Kinetic GFR when the Plasma Creatinine Is Changing Acutely. JASN [Internet]. 2013 May 23;24(6):87788. Available from: http://dx.doi.org/10.1681/ ASN.2012070653

5. Pianta TJ, Endre ZH, Pickering JW, Buckley NA, Peake PW. Kinetic Estimation of GFR Improves Prediction of Dialysis and Recovery after Kidney Transplantation. Remuzzi G, editor. PLoS ONE [Internet]. 2015 May 4;10(5):e0125669. Available from: http://dx.doi.org/10.1371/journal. pone. 0125669

6. O'Sullivan ED, Doyle A. The clinical utility of kinetic glomerular filtration rate. Clin Kidney J [Internet]. 2016 Dec 30;sfw 108. Available from: http://dx.doi.org/10.1093/ $\mathrm{ckj} / \mathrm{sfw} 108$

7. Knaus WA, Draper EA, Wagner DP, Zimmerman JE. APACHE II: a severity of disease classification system. Crit Care Med. 1985 Oct;13(10):818-29.
8. Bouchard J, Macedo E, Soroko S, Chertow GM, Himmelfarb J, Ikizler TA, et al. Comparison of methods for estimating glomerular filtration rate in critically ill patients with acute kidney injury. Nephrology Dialysis Transplantation [Internet]. 2009 Aug 13;25(1):102-7. Available from: http://dx.doi.org/10.1093/ndt/gfp392

9. Shemin D, Bostom AG, Laliberty P, Dworkin LD. Residual renal function and mortality risk in hemodialysis patients. American Journal of Kidney Diseases [Internet]. 2001 Jul;38(1):85-90. Available from: http://dx.doi. org/10.1053/ajkd.2001.25198

10. Daniel WW, Cross C. 2013. Biostatistics: a foundation for analysis in the health sciences (10th ed.). U.S.A.: Wiley.

The study was granted approval by the Ethics Committee of the University of Santo Tomas Institutional Review Board.

(c) Open Access This article is licensed under a Creative Commons Attribution 4.0 International License, which permits use, sharing, adaptation, distribution and reproduction in any medium or format, as long as you give appropriate credit to the original author(s) and the source, provide a link to the Creative Commons license, and indicate if changes were made. The images or other third party material in this article are included in the article's Creative Commons license, unless indicated otherwise in a credit line to the material. If material is not included in the article's Creative Commons license and your intended use is not permitted by statutory regulation or exceeds the permitted use, you will need to obtain permission directly from the copyright holder. To view a copy of this license, visit http://creativecommons.org/licenses/by/4.0/. 